\title{
Enthalpy-Entropy Compensation Reveals Solvent Reorganization as a Driving Force for Supramolecular Encapsulation in Water
}

\author{
Dennis H. Leung, Robert G. Bergman, Kenneth N. Raymond \\ Department of Chemistry, University of California, Berkeley, CA 94720-1460
}

\section{Supporting Information}

\section{Experimental}

General. Unless otherwise noted, reactions and manipulations were carried out under inert atmosphere by using standard Schlenk and vacuum techniques or in a Vacuum Atmospheres inert atmosphere glove box $\left(\mathrm{N}_{2}\right)$ at ambient temperature. ${ }^{1} \mathrm{H}$, ${ }^{13} \mathrm{C}\left\{{ }^{1} \mathrm{H}\right\},{ }^{31} \mathrm{P}\left\{{ }^{1} \mathrm{H}\right\}$, and ${ }^{19} \mathrm{~F}$ spectra were recorded at $400,101,162$, and $376 \mathrm{MHz}$ respectively on a Bruker AVB-400 or AVQ-400 spectrometer. All NMR chemical shifts are reported as $\delta$ in parts per million (ppm). ${ }^{1} \mathrm{H}$ and ${ }^{13} \mathrm{C}\left\{{ }^{1} \mathrm{H}\right\}$ NMR spectra are reported relative to residual solvent. ${ }^{31} \mathrm{P}\left\{{ }^{1} \mathrm{H}\right\}$ NMR spectra are reported relative to trimethyl phosphate as the external standard. ${ }^{19} \mathrm{~F}$ NMR spectra are reported relative to $\mathrm{CFCl}_{3}$ as the external standard. Coupling constants are reported in Hz. Suitable ${ }^{13} \mathrm{C}\left\{{ }^{1} \mathrm{H}\right\}$ NMR spectra of transition metal host-guest complexes could not be obtained after 4000 scans with a pre-scan delay set to 10 seconds. Due to the extensive fluorine coupling, suitable ${ }^{13} \mathrm{C}$ NMR spectra could not be obtained for the fluorinated complexes $3-\mathbf{R}_{\mathbf{F}}$. Infrared (IR) spectra were recorded using a Mattson Instruments Galaxy 3000 Fourier Transform spectrometer or a ThermoNicolet Avatar 370 FT-IR spectrometer equipped with an attenuated total reflectance (ATR) accessory. Elemental analyses (EA) were performed 
by the Microanalytical Laboratory in the College of Chemistry at the University of California, Berkeley. Since an indeterminate amount of solvent remains fixed to the exterior of the cluster, suitable elemental analyses of the host-guest complexes could not be obtained.

All reagents were used as received from commercial suppliers unless otherwise noted. Anhydrous solvents were passed through a column of activated alumina (type A2, size $12 \times 32$, Purify) under nitrogen pressure and sparged with nitrogen before use. ${ }^{1}$ Deuterated solvents were purchased from Cambridge Isotope Laboratories and were either vacuum transferred from $\mathrm{CaH}_{2}$ or degassed by three freeze-pump-thaw cycles and placed over activated $4 \AA$ molecular sieves under inert atmosphere. Water and deuterated water were sparged with nitrogen for thirty minutes to remove oxygen and stored under inert atmosphere. Reactions with trimethylphosphine involved condensation of a measured pressure of gas from a bulb of known volume into a reaction vessel at $-196{ }^{\circ} \mathrm{C}$. Known-volume bulb vacuum transfers were accomplished with a MKS Baratron gauge attached to a high-vacuum line.

The compounds $\mathrm{H}_{4} \mathrm{~L}$ and $\mathrm{Na}_{12}\left[\mathrm{Ga}_{4} \mathrm{~L}_{6}\right]$ were prepared using procedure previously developed by Raymond and coworkers. ${ }^{2-4}$ The compound $\mathrm{Cp}^{*}\left(\mathrm{PMe}_{3}\right) \operatorname{Ir}(\mathrm{Me})(\mathrm{OTf})$ and the carbonyl complexes 2-R were prepared using a procedure developed by Bergman and coworkers. ${ }^{5,6}$ The cationic iridium cis-butene complex $\mathbf{1}$ was prepared as previously reported. ${ }^{7} \mathrm{Cp} * \mathrm{Ir}(\mathrm{CO})_{2}$ was prepared according to literature procedure. ${ }^{8}$ The perfluoroalkyl iridium complexes $\mathrm{Cp} *\left(\mathrm{PMe}_{3}\right) \operatorname{Ir}(\mathrm{I})\left(\mathrm{CF}_{2} \mathrm{CF}_{3}\right), \mathrm{Cp} *\left(\mathrm{PMe}_{3}\right) \operatorname{Ir}(\mathrm{I})\left(\mathrm{CF}_{2} \mathrm{CF}_{2} \mathrm{CF}_{3}\right)$, and $\mathrm{Cp} *\left(\mathrm{PMe}_{3}\right) \operatorname{Ir}(\mathrm{I})\left(\mathrm{CF}\left(\mathrm{CF}_{3}\right)_{2}\right)$ were prepared using procedures developed by Hughes and 
coworkers. ${ }^{9-11}$ For experiments with gaseous reagents, NMR tubes fitted with Teflon stopcocks (J. Young tubes) were used.

$\mathbf{C p} * \operatorname{Ir}(\mathbf{C O})\left(\mathbf{C F}_{3}\right)(\mathbf{I})$. This was prepared according to procedures developed by Hughes and coworkers. ${ }^{9-11}$ In the glove box, a flame-dried Schlenk flask was charged with a magnetic stir bar and a solution of $\mathrm{Cp}^{*} \operatorname{Ir}(\mathrm{CO})_{2}(200 \mathrm{mg}, 0.52 \mathrm{mmol})$ dissolved in $100 \mathrm{~mL} \mathrm{CH}_{2} \mathrm{Cl}_{2}$. The Schlenk flask was removed from the glove box and degassed with three freeze-pump-thaw cycles on a vacuum line before 1 atm $\mathrm{CF}_{3} \mathrm{I}$ was added. The solution was stirred overnight. The flask was brought into the glove box and the solvent was removed in vacuo to give an orange powder. Yield: $196 \mathrm{mg}, 68 \%$. ${ }^{1} \mathrm{H}$ NMR $\left(\mathrm{CD}_{2} \mathrm{Cl}_{2}\right): \delta 2.10\left(\mathrm{~s}, 15 \mathrm{H}, \mathrm{C}_{5}\left(\mathrm{CH}_{3}\right)_{5}\right) .{ }^{19} \mathrm{~F} \mathrm{NMR}\left(\mathrm{CD}_{2} \mathrm{Cl}_{2}\right): \delta-4.6\left(\mathrm{~s}, \mathrm{CF}_{3}\right) . \quad \mathrm{IR}(\mathrm{ATR}):$ $v_{\mathrm{CO}}=2020 \mathrm{~cm}^{-1}$. Anal. Cald for $\mathrm{C}_{12} \mathrm{H}_{15} \mathrm{~F}_{3}$ IOIr: C, 26.14; H, 2.74. Found: C, 26.17; H, 2.68 .

Cp*(PMe $) \operatorname{Ir}(\mathbf{I})\left(\mathbf{C F}_{3}\right)$. This was prepared in analogy to procedures developed by Hughes and coworkers. ${ }^{9-11}$ In the glove box, a flame-dried Schlenk flask was charged with a magnetic stir bar and a solution of $\mathrm{Cp}^{*} \operatorname{Ir}(\mathrm{CO})\left(\mathrm{CF}_{3}\right)(\mathrm{I})(100 \mathrm{mg}, 0.18 \mathrm{mmol})$ dissolved in $10 \mathrm{~mL} \mathrm{CH}_{2} \mathrm{Cl}_{2}$. The Schlenk flask was removed from the glove box and degassed with three freeze-pump-thaw cycles on a vacuum line. Using a known-volume bulb, a stochiometric amount of $\mathrm{PMe}_{3}$ was condensed into the flask at $-196{ }^{\circ} \mathrm{C}$ using liquid nitrogen. The flask was warmed to room temperature and stirred for 1 hour. The solution turned yellow and white precipitate formed. The flask was brought back into the glove box and the solution was filtered to give a yellow filtrate. The solvent was removed from the filtrate to give a yellow solid. Yield: $36 \mathrm{mg}, 33 \% .{ }^{1} \mathrm{H}$ NMR $\left(\mathrm{CD}_{2} \mathrm{Cl}_{2}\right): \delta 1.87\left(\mathrm{~d}, J_{\mathrm{P}-\mathrm{H}}=2.0 \mathrm{~Hz}, 15 \mathrm{H}, \mathrm{C}_{5}\left(\mathrm{CH}_{3}\right)_{5}\right), 1.71\left(\mathrm{~d}, J_{\mathrm{P}-\mathrm{H}}=10.8 \mathrm{~Hz}, 9 \mathrm{H}\right.$, 
$\left.\mathrm{P}\left(\mathrm{CH}_{3}\right)_{3}\right) .{ }^{31} \mathrm{P}\left\{{ }^{1} \mathrm{H}\right\} \mathrm{NMR}\left(\mathrm{CD}_{2} \mathrm{Cl}_{2}\right): \delta-39.39\left(\mathrm{~s}, P\left(\mathrm{CH}_{3}\right)_{3}\right) .{ }^{19} \mathrm{~F} \mathrm{NMR}\left(\mathrm{CD}_{2} \mathrm{Cl}_{2}\right): \delta-3.9(\mathrm{~s}$, $\mathrm{CF}_{3}$ ). Anal. Cald for $\mathrm{C}_{14} \mathrm{H}_{24} \mathrm{~F}_{3}$ IPIr: C, 28.05; H, 4.04. Found: C, 28.16; H, 4.07.

$\left[\mathrm{Cp}^{*}\left(\mathrm{PMe}_{3}\right) \operatorname{Ir}(\mathbf{C O})\left(\mathrm{CF}_{3}\right)\right][\mathrm{OTf}]\left(3-\mathrm{Me}_{\mathrm{F}}\right)$. In the glove box, a Schlenk flask was charged with a magnetic stir bar and a solution of $\mathrm{Cp} *\left(\mathrm{PMe}_{3}\right) \operatorname{Ir}(\mathrm{I})\left(\mathrm{CF}_{3}\right)(100 \mathrm{mg}, 0.17$ mmol) dissolved in $10 \mathrm{~mL} \mathrm{CH}_{2} \mathrm{Cl}_{2}$. AgOTf (52 mg, $0.20 \mathrm{mmol}$ ) was added as a solid and precipitate immediately formed. The flask was wrapped in aluminum foil, taken out of the glove box, and attached to a vacuum line. The solution was degassed with three freeze-pump-thaw cycles and $1 \mathrm{~atm} \mathrm{CO}$ was added. The solution was stirred overnight before being brought back into the glove box. The solution was filtered through Celite and the solvent was removed from the colorless filtrate in vacuo to leave a gray solid. Yield: $89 \mathrm{mg}, 82 \% .{ }^{1} \mathrm{H}$ NMR $\left(\mathrm{CD}_{2} \mathrm{Cl}_{2}\right): \delta 2.14\left(\mathrm{~d}, J_{\mathrm{P}-\mathrm{H}}=2.0 \mathrm{~Hz}, 15 \mathrm{H}, \mathrm{C}_{5}\left(\mathrm{CH}_{3}\right)_{5}\right), 1.90$ $\left(\mathrm{d}, J_{\mathrm{P}-\mathrm{H}}=11.6 \mathrm{~Hz}, 9 \mathrm{H}, \mathrm{P}\left(\mathrm{CH}_{3}\right)_{3}\right) .{ }^{31} \mathrm{P}\left\{{ }^{1} \mathrm{H}\right\} \mathrm{NMR}\left(\mathrm{CD}_{2} \mathrm{Cl}_{2}\right): \delta-37.6\left(\mathrm{q}, J_{\mathrm{P}-\mathrm{F}}=4.9 \mathrm{~Hz}\right.$, $\left.P\left(\mathrm{CH}_{3}\right)_{3}\right) .{ }^{19} \mathrm{~F} \mathrm{NMR}\left(\mathrm{CD}_{2} \mathrm{Cl}_{2}\right): \delta-5.3\left(\mathrm{~s}, \mathrm{CF}_{3}\right),-78.1\left(\mathrm{~s}, \mathrm{OSO}_{2} \mathrm{CF}_{3}\right)$. IR $(\mathrm{ATR}): v_{\mathrm{CO}}=$ $2059 \mathrm{~cm}^{-1}$. Anal. Cald for $\mathrm{C}_{16} \mathrm{H}_{24} \mathrm{SF}_{6} \mathrm{O}_{4} \mathrm{PIr}$ : C, 29.58; H, 3.72; S, 4.94. Found: C, 29.28; H, 3.68; S, 5.06.

$\left[\mathrm{Cp}^{*}\left(\mathrm{PMe}_{3}\right) \operatorname{Ir}(\mathbf{C O})\left(\mathrm{CF}_{2} \mathbf{C F}_{3}\right)\right][\mathrm{OTf}]\left(3-\mathbf{E t}_{\mathbf{F}}\right)$. In the glove box, a Schlenk flask was charged with a magnetic stir bar and a solution of $\mathrm{Cp} *\left(\mathrm{PMe}_{3}\right) \operatorname{Ir}(\mathrm{I})\left(\mathrm{CF}_{2} \mathrm{CF}_{3}\right)(100 \mathrm{mg}$, $0.15 \mathrm{mmol})$ dissolved in $10 \mathrm{~mL} \mathrm{CH}_{2} \mathrm{Cl}_{2}$. AgOTf ( $\left.47 \mathrm{mg}, 0.18 \mathrm{mmol}\right)$ was added as a solid and precipitate immediately formed. The flask was wrapped in aluminum foil, taken out of the glove box, and attached to a vacuum line. The solution was degassed with three freeze-pump-thaw cycles and $1 \mathrm{~atm} \mathrm{CO}$ was added. The solution was stirred overnight before being brought back into the glove box. The solution was filtered through Celite and the solvent was removed from the colorless filtrate in vacuo to leave a 
gray solid. Yield: $94 \mathrm{mg}, 87 \%$. ${ }^{1} \mathrm{H}$ NMR $\left(\mathrm{CD}_{2} \mathrm{Cl}_{2}\right): \delta 2.12\left(\mathrm{~d}, J_{\mathrm{P}-\mathrm{H}}=2.0 \mathrm{~Hz}, 15 \mathrm{H}\right.$, $\left.\mathrm{C}_{5}\left(\mathrm{CH}_{3}\right)_{5}\right), 1.92\left(\mathrm{~d}, J_{\mathrm{P}-\mathrm{H}}=11.6 \mathrm{~Hz}, 9 \mathrm{H}, \mathrm{P}\left(\mathrm{CH}_{3}\right)_{3}\right) .{ }^{31} \mathrm{P}\left\{{ }^{1} \mathrm{H}\right\} \mathrm{NMR}\left(\mathrm{CD}_{2} \mathrm{Cl}_{2}\right): \delta-37.6(\mathrm{dd}$, $\left.J_{\mathrm{P}-\mathrm{F}}=43.7 \mathrm{~Hz}, J_{\mathrm{P}-\mathrm{F}}=3.2 \mathrm{~Hz}, P\left(\mathrm{CH}_{3}\right)_{3}\right) .{ }^{19} \mathrm{~F} \mathrm{NMR}\left(\mathrm{CD}_{2} \mathrm{Cl}_{2}\right): \delta-71.0\left(\mathrm{~d}, J_{\mathrm{F}-\mathrm{F}}=274.5 \mathrm{~Hz}\right.$, $\left.\mathrm{C} \alpha F_{\mathrm{B}}\right),-71.1\left(\mathrm{~d}, J_{\mathrm{F}-\mathrm{F}}=274.5 \mathrm{~Hz}, \mathrm{C} \alpha F_{\mathrm{B}}\right),-78.1\left(\mathrm{~s}, \mathrm{OSO}_{2} \mathrm{CF}_{3}\right),-83.1\left(\mathrm{~s}, \mathrm{CF}_{3}\right)$. IR (ATR): $v_{\mathrm{CO}}=2049 \mathrm{~cm}^{-1}$. Anal. Cald for $\mathrm{C}_{17} \mathrm{H}_{24} \mathrm{SF}_{8} \mathrm{O}_{4} \mathrm{PIr}$ : C, 29.19; H, 3.46; S, 4.58. Found: C, $28.78 ; \mathrm{H}, 3.23 ; \mathrm{S}, 4.66$.

[Cp*(PMe $\left.) \operatorname{Ir}(\mathbf{C O})\left(\mathrm{CF}_{2} \mathrm{CF}_{2} \mathrm{CF}_{3}\right)\right][\mathrm{OTf}]$ (3-PrF $)$. In the glove box, a Schlenk flask was charged with a magnetic stir bar and a solution of $\mathrm{Cp} *\left(\mathrm{PMe}_{3}\right) \operatorname{Ir}(\mathrm{I})\left(\mathrm{CF}_{2} \mathrm{CF}_{2} \mathrm{CF}_{3}\right)$

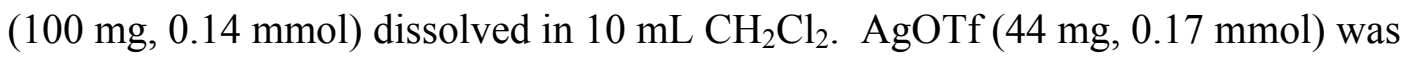
added as a solid and precipitate immediately formed. The flask was wrapped in aluminum foil, taken out of the glove box, and attached to a vacuum line. The solution was degassed with three freeze-pump-thaw cycles and $1 \mathrm{~atm} \mathrm{CO}$ was added. The solution was stirred overnight before being brought back into the glove box. The solution was filtered through Celite and the solvent was removed from the colorless filtrate in vacuo to leave a gray solid. Yield: $96 \mathrm{mg}, 90 \% .{ }^{1} \mathrm{H}$ NMR $\left(\mathrm{CD}_{2} \mathrm{Cl}_{2}\right): \delta 2.21\left(\mathrm{~d}, J_{\mathrm{P}-\mathrm{H}}=\right.$ $\left.2.4 \mathrm{~Hz}, 15 \mathrm{H}, \mathrm{C}_{5}\left(\mathrm{CH}_{3}\right)_{5}\right), 1.93\left(\mathrm{~d}, J_{\mathrm{P}-\mathrm{H}}=11.6 \mathrm{~Hz}, 9 \mathrm{H}, \mathrm{P}\left(\mathrm{CH}_{3}\right)_{3}\right) .{ }^{31} \mathrm{P}\left\{{ }^{1} \mathrm{H}\right\} \mathrm{NMR}\left(\mathrm{CD}_{2} \mathrm{Cl}_{2}\right)$ : $\delta-34.2\left(\mathrm{~d}, J_{\mathrm{P}-\mathrm{F}}=45.4 \mathrm{~Hz}, P\left(\mathrm{CH}_{3}\right)_{3}\right) .{ }^{19} \mathrm{~F} \mathrm{NMR}\left(\mathrm{CD}_{2} \mathrm{Cl}_{2}\right): \delta-67.9\left(\mathrm{~d}, J_{\mathrm{F}-\mathrm{F}}=289.5 \mathrm{~Hz}\right.$, $\left.\left.\mathrm{C} \alpha F_{\mathrm{A}}\right),-74.0\left(\mathrm{~d}, J_{\mathrm{F}-\mathrm{F}}=285.8 \mathrm{~Hz}, \mathrm{C} \alpha F_{\mathrm{B}}\right),-78.1\left(\mathrm{~s}, \mathrm{OSO}_{2} \mathrm{CF}_{3}\right),-78.3(\mathrm{~m}, \mathrm{CF})_{3}\right),-115.1(\mathrm{~m}$, $\mathrm{C}_{\beta} F_{2}$ ). IR (ATR): $v_{\mathrm{CO}}=2059 \mathrm{~cm}^{-1}$. Anal. Cald for $\mathrm{C}_{18} \mathrm{H}_{24} \mathrm{SF}_{10} \mathrm{O}_{4} \mathrm{PIr}: \mathrm{C}, 28.84 ; \mathrm{H}, 3.23$; S, 4.28. Found: C, 28.50; H, 2.97; S, 4.42 .

[Cp*(PMe 3$\left.) \operatorname{Ir}(\mathbf{C O})\left(\mathbf{C F}\left(\mathrm{CF}_{3}\right)_{2}\right)\right][\mathbf{O T f}]\left({ }^{3}-{ }^{\mathrm{i}} \mathbf{P r}_{\mathbf{F}}\right)$. In the glove box, a Schlenk flask was charged with a magnetic stir bar and a solution of $\mathrm{Cp} *\left(\mathrm{PMe}_{3}\right) \operatorname{Ir}(\mathrm{I})\left(\mathrm{CF}\left(\mathrm{CF}_{3}\right)_{2}\right)(100$

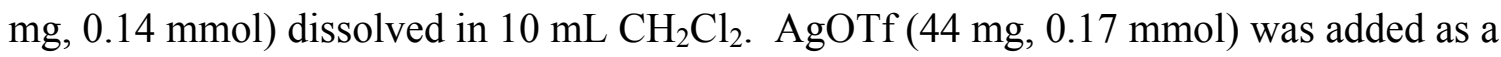


solid and precipitate immediately formed. The flask was wrapped in aluminum foil, taken out of the glove box, and attached to a vacuum line. The solution was degassed with three freeze-pump-thaw cycles and $1 \mathrm{~atm} \mathrm{CO}$ was added. The solution was stirred overnight before being brought back into the glove box. The solution was filtered through Celite and the solvent was removed from the colorless filtrate in vacuo to leave a gray solid. Yield: $90 \mathrm{mg}, 84 \% .{ }^{1} \mathrm{H}$ NMR $\left(\mathrm{CD}_{2} \mathrm{Cl}_{2}\right): \delta 2.09\left(\mathrm{~d}, J_{\mathrm{P}-\mathrm{H}}=2.0 \mathrm{~Hz}, 15 \mathrm{H}\right.$, $\left.\mathrm{C}_{5}\left(\mathrm{CH}_{3}\right)_{5}\right), 1.88\left(\mathrm{dd}, J_{\mathrm{P}-\mathrm{H}}=11.2 \mathrm{~Hz}, J_{\mathrm{F}-\mathrm{H}}=2.0 \mathrm{~Hz}, 9 \mathrm{H}, \mathrm{P}\left(\mathrm{CH}_{3}\right)_{3}\right) .{ }^{31} \mathrm{P}\left\{{ }^{1} \mathrm{H}\right\} \mathrm{NMR}$ $\left(\mathrm{CD}_{2} \mathrm{Cl}_{2}\right): \delta-35.2\left(\right.$ br m, $\left.P\left(\mathrm{CH}_{3}\right)_{3}\right) .{ }^{19} \mathrm{~F} \mathrm{NMR}\left(\mathrm{CD}_{2} \mathrm{Cl}_{2}\right): \delta-69.5\left(\mathrm{~s}, \mathrm{CF}\left(\mathrm{CF}_{3}\right)_{2}\right),-69.9(\mathrm{~s}$, $\left.\mathrm{CF}\left(\mathrm{CF}_{3}\right)_{2}\right),-78.1\left(\mathrm{~s}, \mathrm{OSO}_{2} \mathrm{CF}_{3}\right),-154.5\left(\mathrm{br}, \mathrm{CF}\left(\mathrm{CF}_{3}\right)_{2}\right)$. IR (ATR): $v_{\mathrm{CO}}=2052 \mathrm{~cm}^{-1}$. Anal. Cald for $\mathrm{C}_{18} \mathrm{H}_{24} \mathrm{SF}_{10} \mathrm{O}_{4} \mathrm{PIr}: \mathrm{C}, 28.84 ; \mathrm{H}, 3.23 ; \mathrm{S}, 4.28$. Found: C, 28.50; H, 3.20, S, 4.45 .

$\mathbf{N a}_{11}\left[3-\mathbf{M e}_{\mathbf{F}} \subset \mathbf{G a}_{4} \mathbf{L}_{6}\right]$. In the glove box, an NMR tube was charged with 3-Me $\mathbf{e}_{\mathbf{F}}$ $\left(\sim 3 \mathrm{mg}, 4.6 \times 10^{-3} \mathrm{mmol}\right)$ as a solid. This was dissolved in $0.5 \mathrm{~mL} \mathrm{D}_{2} \mathrm{O}$ to give a pale yellow solution. A stoichiometric amount of $\mathrm{Na}_{12}\left[\mathrm{Ga}_{4} \mathrm{~L}_{6}\right]\left(\sim 16 \mathrm{mg}, 4.6 \times 10^{-3} \mathrm{mmol}\right)$ was added as a solid to form a bright yellow solution. The NMR tube was then removed from the glove box and flame-sealed under static vacuum on a vacuum line. Two diastereomeric host-guest complexes A and B were observed with a dr of 57:43 which were isolated upon removal of solvent in vacuo. ${ }^{1} \mathrm{H}$ NMR $\left(\mathrm{D}_{2} \mathrm{O}\right): \delta 8.41$ (br, aryl), 8.09 (br, aryl), 7.67 (d, $J=8.5 \mathrm{~Hz}$, aryl), 7.53 (br, aryl), 7.41 (d, $J=7.5 \mathrm{~Hz}, \operatorname{aryl}), 7.35$ (d, $J=$ $8.0 \mathrm{~Hz}$, aryl), 7.04 (br, aryl), 6.92 (m, aryl), 6.77 (m, aryl), 6.63 (m, aryl), 0.00 (d, J=2.0 $\left.\mathrm{Hz}, 15 \mathrm{H}, \mathrm{C}_{5}\left(\mathrm{CH}_{3}\right)_{5} \mathrm{~B}\right),-0.05\left(\mathrm{~d}, J=2.0 \mathrm{~Hz}, 15 \mathrm{H}, \mathrm{C}_{5}\left(\mathrm{CH}_{3}\right)_{5} \mathrm{~A}\right),-0.49(\mathrm{~d}, J=11.5 \mathrm{~Hz}, 9 \mathrm{H}$, $\left.\mathrm{P}\left(\mathrm{CH}_{3}\right)_{3} \mathrm{~A}\right),-0.70\left(\mathrm{~d}, J=11.5 \mathrm{~Hz}, 9 \mathrm{H}, \mathrm{P}\left(\mathrm{CH}_{3}\right)_{3} \mathrm{~B}\right) .{ }^{31} \mathrm{P}\left\{{ }^{1} \mathrm{H}\right\} \mathrm{NMR}\left(\mathrm{CD}_{2} \mathrm{Cl}_{2}\right): \delta-41.6(\mathrm{br}$ 
m, $\left.P\left(\mathrm{CH}_{3}\right)_{3} \mathrm{~B}\right),-44.7$ (br m, $\left.P\left(\mathrm{CH}_{3}\right)_{3} \mathrm{~A}\right) .{ }^{19} \mathrm{~F} \mathrm{NMR}\left(\mathrm{CD}_{2} \mathrm{Cl}_{2}\right): \delta-7.6\left(\mathrm{~s}, \mathrm{CF}_{3} \mathrm{~A}\right),-7.7(\mathrm{~s}$, $\left.\mathrm{CF}_{3} \mathrm{~B}\right),-78.1\left(\mathrm{~s}, \mathrm{OSO}_{2} \mathrm{CF}_{3}\right)$. IR (ATR): $v_{\mathrm{CO}}=2053 \mathrm{~cm}^{-1}$.

$\mathbf{N a} \mathbf{a}_{11}\left[3-\mathbf{E} \mathbf{t}_{\mathbf{F}} \subset \mathbf{G} \mathbf{a}_{\mathbf{4}} \mathbf{L}_{\mathbf{6}}\right]$. In the glove box, an NMR tube was charged with 3-Et $\left(\sim 3 \mathrm{mg}, 4.3 \times 10^{-3} \mathrm{mmol}\right)$ as a solid. This was dissolved in $0.5 \mathrm{~mL} \mathrm{D}_{2} \mathrm{O}$ to give a pale yellow solution. A stoichiometric amount of $\mathrm{Na}_{12}\left[\mathrm{Ga}_{4} \mathrm{~L}_{6}\right]\left(\sim 15 \mathrm{mg}, 4.3 \times 10^{-3} \mathrm{mmol}\right)$ was added as a solid to form a bright yellow solution. The NMR tube was then removed from the glove box and flame-sealed under static vacuum on a vacuum line. Two diastereomeric host-guest complexes A and B were observed with a dr of 51:49 which were isolated upon removal of solvent in vacuo. ${ }^{1} \mathrm{H}$ NMR $\left(\mathrm{D}_{2} \mathrm{O}\right): \delta 8.47(\mathrm{~m}$, aryl $), 8.37$ $(\mathrm{d}, J=8.0 \mathrm{~Hz}, \operatorname{aryl}), 8.17(\mathrm{~d}, J=7.5 \mathrm{~Hz}, \operatorname{aryl}), 8.12(\mathrm{~d}, J=7.5 \mathrm{~Hz}, \operatorname{aryl}), 8.07$ (d, $J=7.5$ Hz, aryl), $7.97(\mathrm{~m}$, aryl), $7.82(\mathrm{~d}, J=8.5 \mathrm{~Hz}$, aryl), $7.72(\mathrm{~m}$, aryl $), 7.64(\mathrm{~d}, J=8.5 \mathrm{~Hz}$, aryl), $7.57(\mathrm{~m}, \operatorname{aryl}), 7.52(\mathrm{~d}, J=8.5 \mathrm{~Hz}, \operatorname{aryl}), 7.48(\mathrm{~m}, \operatorname{aryl}), 7.44(\mathrm{~m}, \operatorname{aryl}), 7.42(\mathrm{~m}$, aryl), $7.39(\mathrm{~m}, \operatorname{aryl}), 7.37$ (m, aryl), $7.35(\mathrm{~m}, \operatorname{aryl}), 7.20(\mathrm{~d}, J=8.5 \mathrm{~Hz}, \operatorname{aryl}), 7.02(\mathrm{~m}$, aryl), 6.93 (m, aryl), 6.87 (m, aryl), 6.85 (m, aryl), 6.83 (m, aryl), 6.81 (br, aryl), 6.80 (m, aryl), 6.78 (br, aryl), $6.76(\mathrm{~m}$, aryl), $6.75(\mathrm{~m}, \operatorname{aryl}), 6.73(\mathrm{~m}$, aryl $), 6.71$ (br, aryl), 6.69 (m, aryl), 6.67 (m, aryl), 6.65 (br, aryl), 6.64 (br, aryl), 6.59 (m, aryl), 6.57 (m, $\operatorname{aryl}), 0.16\left(\mathrm{~d}, J=2.0 \mathrm{~Hz}, 15 \mathrm{H}, \mathrm{C}_{5}\left(\mathrm{CH}_{3}\right)_{5} \mathrm{~A}\right), 0.10\left(\mathrm{~d}, J=2.0 \mathrm{~Hz}, 15 \mathrm{H}, \mathrm{C}_{5}\left(\mathrm{CH}_{3}\right)_{5} \mathrm{~B}\right),-0.25$ $\left(\mathrm{d}, J=11.5 \mathrm{~Hz}, 9 \mathrm{H}, \mathrm{P}\left(\mathrm{CH}_{3}\right)_{3} \mathrm{~B}\right),-0.33\left(\mathrm{~d}, J=11.0 \mathrm{~Hz}, 9 \mathrm{H}, \mathrm{P}\left(\mathrm{CH}_{3}\right)_{3} \mathrm{~A}\right) .{ }^{31} \mathrm{P}\left\{{ }^{1} \mathrm{H}\right\} \mathrm{NMR}$ $\left(\mathrm{CD}_{2} \mathrm{Cl}_{2}\right): \delta-42.1$ (br m, $\left.P\left(\mathrm{CH}_{3}\right)_{3} \mathrm{~A}\right),-41.4$ (br m, $\left.P\left(\mathrm{CH}_{3}\right)_{3} \mathrm{~B}\right) .{ }^{19} \mathrm{~F} \mathrm{NMR}\left(\mathrm{CD}_{2} \mathrm{Cl}_{2}\right): \delta-$ $70.5\left(\mathrm{~d}, J_{\mathrm{F}-\mathrm{F}}=278.2 \mathrm{~Hz}, \mathrm{C} \alpha F_{\mathrm{B}}\right),-72.2\left(\mathrm{~d}, J_{\mathrm{F}-\mathrm{F}}=293.3 \mathrm{~Hz}, \mathrm{C} \alpha F_{\mathrm{B}}\right),-78.1\left(\mathrm{~s}, \mathrm{OSO}_{2} \mathrm{CF}_{3}\right),-$ $80.1\left(\mathrm{~s}, \mathrm{CF}_{3} \mathrm{~B}\right), 81.0\left(\mathrm{~s}, \mathrm{CF}_{3} \mathrm{~A}\right)$. IR (ATR): $v_{\mathrm{CO}}=2060 \mathrm{~cm}^{-1}$.

$\mathbf{N a}_{11}\left[3-{ }^{\mathrm{i}} \mathbf{P r}_{\mathbf{F}} \subset \mathbf{G a}_{\mathbf{4}} \mathbf{L}_{6}\right]$. In the glove box, an NMR tube was charged with $3-{ }^{\mathrm{i}} \mathbf{P r}_{\mathbf{F}}$ $\left(\sim 3 \mathrm{mg}, 4.0 \times 10^{-3} \mathrm{mmol}\right)$ as a solid. This was dissolved in $0.5 \mathrm{~mL} \mathrm{D}_{2} \mathrm{O}$ to give a pale 
yellow solution. A stoichiometric amount of $\mathrm{Na}_{12}\left[\mathrm{Ga}_{4} \mathrm{~L}_{6}\right]\left(\sim 14 \mathrm{mg}, 4.0 \times 10^{-3} \mathrm{mmol}\right)$ was added as a solid to form a bright yellow solution. The NMR tube was then removed from the glove box and flame-sealed under static vacuum on a vacuum line. Two diastereomeric host-guest complexes A and B were observed with a dr of 86:14 which were isolated upon removal of solvent in vacuo. ${ }^{1} \mathrm{H}$ NMR $\left(\mathrm{D}_{2} \mathrm{O}\right): \delta 8.36(\mathrm{~m}$, aryl $), 8.32$ (d, $J=8.0 \mathrm{~Hz}$, aryl), 8.30 (d, $J=8.5 \mathrm{~Hz}$, aryl), $8.24(\mathrm{~d}, J=8.0 \mathrm{~Hz}, \operatorname{aryl}), 8.20$ (d, $J=7.5$ $\mathrm{Hz}, \operatorname{aryl}), 8.04(\mathrm{~d}, J=7.0 \mathrm{~Hz}$, aryl), $8.01(\mathrm{~d}, J=8.0 \mathrm{~Hz}, \operatorname{aryl}), 7.64(\mathrm{~d}, J=8.5 \mathrm{~Hz}$, aryl), $7.59(\mathrm{~d}, J=8.5 \mathrm{~Hz}, \operatorname{aryl}), 7.47(\mathrm{~m}$, aryl), $7.41(\mathrm{~m}, \operatorname{aryl}), 7.20(\mathrm{~d}, J=8.5 \mathrm{~Hz}, \operatorname{aryl}), 6.87$ (m, aryl), $6.85(\mathrm{~m}, \operatorname{aryl}), 6.82(\mathrm{~m}, \operatorname{aryl}), 6.81(\mathrm{~m}, \operatorname{aryl}), 6.76(\mathrm{br} \mathrm{m}, \operatorname{aryl}), 6.71(\mathrm{br}, \operatorname{aryl})$, 6.69 (br, aryl), 6.67 (br, aryl), 6.65 (br, aryl), 6.64 (br, aryl), 6.62 (br, aryl), 6.60 (m, aryl), $0.22\left(\mathrm{~d}, J=2.0 \mathrm{~Hz}, 15 \mathrm{H}, \mathrm{C}_{5}\left(\mathrm{CH}_{3}\right)_{5} \mathrm{~A}\right), 0.20\left(\mathrm{~d}, J=2.0 \mathrm{~Hz}, 15 \mathrm{H}, \mathrm{C}_{5}\left(\mathrm{CH}_{3}\right)_{5} \mathrm{~B}\right),-0.25$ (d, $J=11.5 \mathrm{~Hz}, 9 \mathrm{H}, \mathrm{P}\left(\mathrm{CH}_{3}\right)_{3} \mathrm{~A}$ and B). ${ }^{31} \mathrm{P}\left\{{ }^{1} \mathrm{H}\right\} \mathrm{NMR}\left(\mathrm{CD}_{2} \mathrm{Cl}_{2}\right): \delta-42.4\left(\right.$ br m, $P\left(\mathrm{CH}_{3}\right)_{3}$ A), $-42.8\left(\mathrm{br} \mathrm{m}, P\left(\mathrm{CH}_{3}\right)_{3} \mathrm{~B}\right) .{ }^{19} \mathrm{~F} \mathrm{NMR}\left(\mathrm{CD}_{2} \mathrm{Cl}_{2}\right): \delta-65.7\left(\mathrm{br}, \mathrm{CF}\left(\mathrm{CF}_{3}\right)_{2} \mathrm{~B}\right),-69.4(\mathrm{br}$, $\left.\mathrm{CF}\left(\mathrm{CF}_{3}\right)_{2} \mathrm{~A}\right),-69.7\left(\mathrm{br}, \mathrm{CF}\left(\mathrm{CF}_{3}\right)_{2} \mathrm{~A}\right),-70.9\left(\mathrm{br}, \mathrm{CF}\left(\mathrm{CF}_{3}\right)_{2} \mathrm{~B}\right),-78.1\left(\mathrm{~s}, \mathrm{OSO}_{2} \mathrm{CF}_{3}\right),-158.4$ (br m, $\mathrm{CF}\left(\mathrm{CF}_{3}\right)_{2} \mathrm{~A}$ and B). IR (ATR): $v_{\mathrm{CO}}=2060 \mathrm{~cm}^{-1}$.

General Procedure for van't Hoff Measurements. Stock solutions of the appropriate encapsulated iridium species (with $10 \mathrm{mM}$ iridium species and $10 \mathrm{mM}$ $\left.\mathrm{Na}_{12}\left[\mathrm{Ga}_{4} \mathrm{~L}_{6}\right]\right)$ were prepared with the appropriate solvent $\left(\mathrm{D}_{2} \mathrm{O}\right.$ buffered with $100 \mathrm{mM}$ TRIS/DOTf at $\mathrm{pD}=8.0, \mathrm{CD}_{3} \mathrm{OD}$, or DMSO- $d_{6}$ ) at room temperature. The $\mathrm{pD}$ of the solution was not corrected at different temperatures. Dioxane $(2 \mu \mathrm{L})$ was added as an internal standard. The reaction was monitored immediately by ${ }^{1} \mathrm{H}$ NMR at a temperature of $24{ }^{\circ} \mathrm{C}$. The concentration of the encapsulated iridium guest was monitored using 8 scans with a delay time of 4 seconds and a $90^{\circ}$ pulse of $13.5 \mu$ sec for each time point. 
Data points were taken at increasing temperature intervals of $10{ }^{\circ} \mathrm{C}$, allowing the sample to equilibrate for $15 \mathrm{~min}$ at each temperature until no further change in guest concentration was observed. The thermodynamic data were plotted on a van't Hoff graph with the observed equilibria constants vs. 1/T and the data was fit using a linear least squares regression. Error analyses for the van't Hoff plots were performed on the slope and intercept of the linear fit.

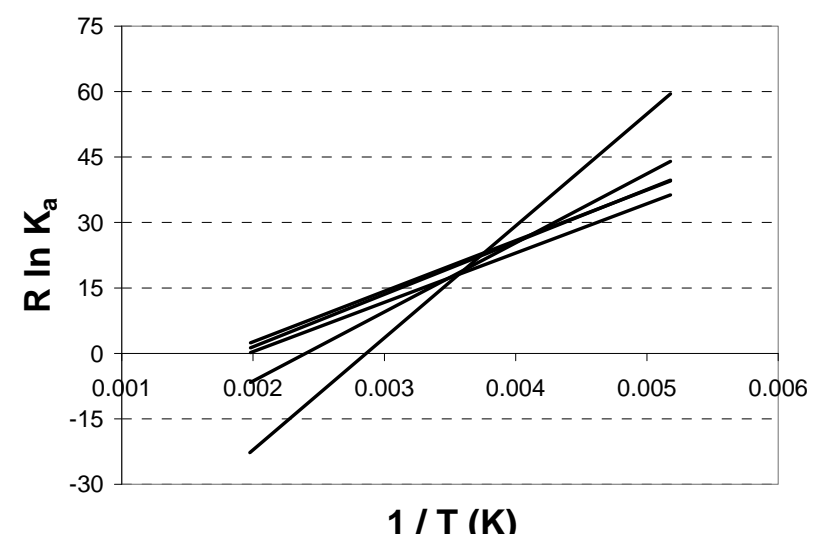

Figure S-1. Plot of van't Hoff slopes for the encapsulation of iridium species 2-R and 3$\mathbf{R}_{\mathbf{F}}$ by $\mathrm{Na}_{12}\left[\mathrm{Ga}_{4} \mathrm{~L}_{6}\right]$ in $\mathrm{CD}_{3} \mathrm{OD}$. The iridium species meet at a common intersection point at $1 / \mathrm{T}_{\mathrm{c}}$.

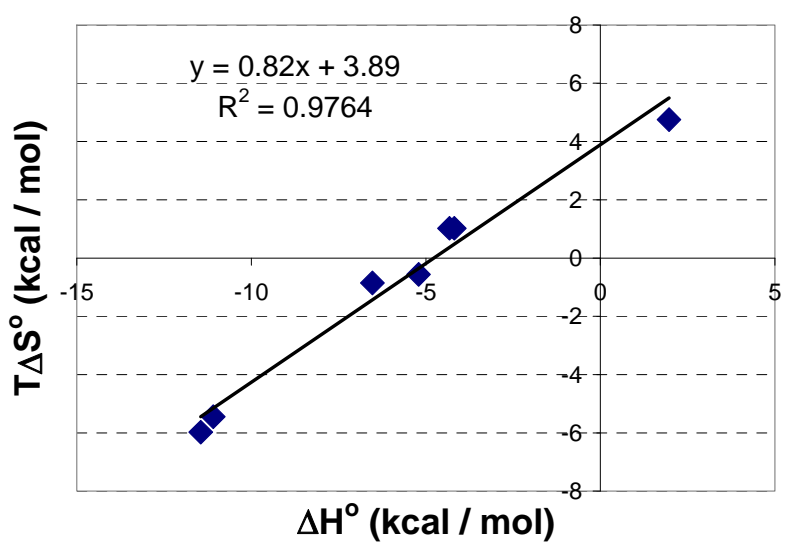

Figure S-2. Plot of $\mathrm{T} \Delta \mathrm{S}^{\circ}$ versus $\Delta \mathrm{H}^{\circ}(\mathrm{T}=298 \mathrm{~K})$ for the encapsulation of iridium species 2-R and 3-R $\mathbf{R}_{\mathbf{F}}$ (not including $3-{ }^{\mathrm{i}} \mathbf{P r}_{\mathbf{F}}$ ) by $\mathrm{Na}_{12}\left[\mathrm{Ga}_{4} \mathrm{~L}_{6}\right]$ in $\mathrm{D}_{2} \mathrm{O}$ buffered with TRIS/DOTf $(100.0 \mathrm{mM})$ at $\mathrm{pD}=8.0$ 


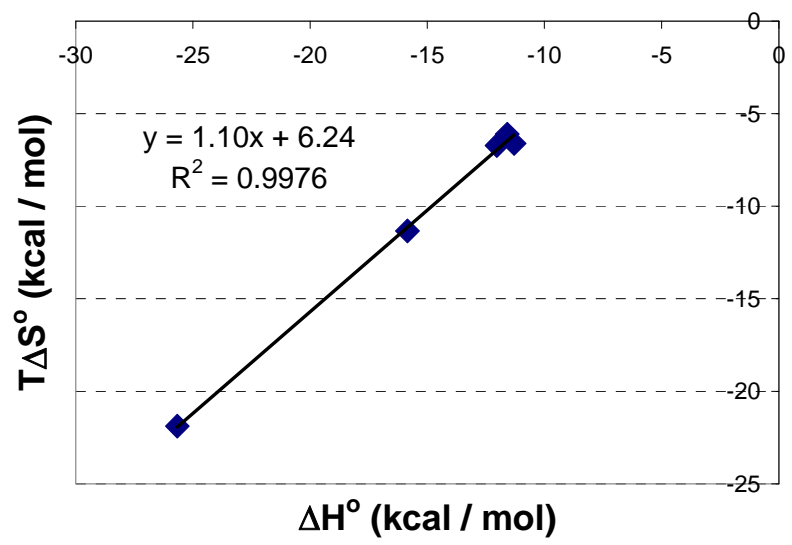

Figure S-3. Plot of $\mathrm{T} \Delta \mathrm{S}^{\circ}$ versus $\Delta \mathrm{H}^{\circ}(\mathrm{T}=298 \mathrm{~K})$ for the encapsulation of iridium species 2-R and 3-R $\mathbf{R}_{\mathbf{F}}$ by $\mathrm{Na}_{12}\left[\mathrm{Ga}_{4} \mathrm{~L}_{6}\right]$ in $\mathrm{CD}_{3} \mathrm{OD}$.

\section{References}

(1) Alaimo, P. J.; Peters, D. W.; Arnold, J.; Bergman, R. G. J. Chem. Educ. 2001, 78, 64 .

(2) Caulder, D. L.; Powers, R. E.; Parac, T. N.; Raymond, K. N. Angew.

Chem. Int. Ed. 1998, 37, 1840-1843.

(3) Caulder, D. L.; Raymond, K. N. Acc. Chem. Res. 1999, 32, 975-982.

(4) Caulder, D. L.; Bruckner, C.; Powers, R. E.; Konig, S.; Parac, T. N.;

Leary, J. A.; Raymond, K. N. J. Am. Chem. Soc. 2001, 123, 8893-8938.

(5) Burger, P.; Bergman, R. G. J. Am. Chem. Soc. 1993, 115, 10462-10463.

(6) Alaimo, P. J.; Arndsten, B. A.; Bergman, R. G. Organometallics 2000, 2000, 2130-2143.

(7) Leung, D. H.; Bergman, R. G.; Raymond, K. N. J. Am. Chem. Soc. 2006, 128, 9781-9797.

(8) Ball, R. G.; Graham, W. A. G.; Heinekey, D. M.; Hoyano, J. K.;

McMaster, A. D.; Mattson, B. M.; Michel, S. T. Inorg. Chem. 1990, 29, 2023-2025.

(9) Hughes, R. P.; Lindner, D. C.; Rheingold, A. L.; Liable-Sands, L. M. J. Am. Chem. Soc. 1997, 119, 11544-11545.

(10) Hughes, R. P.; Smith, J. M.; Liable-Sands, L. M.; Concolino, T. E.; Lam, K.-C.; Incarvito, C.; Rheingold, A. L. J. Chem. Soc., Dalton Trans. 2000, 873-880.

(11) Hughes, R. P.; Zhang, D.; Ward, A. J.; Zakharov, L. N.; Rheingold, A. L. J. Am. Chem. Soc. 2004, 126, 6169-6178. 